\title{
Energy Conservation in Wireless Sensor Networks: A Review
}

\author{
Bandana Bhatia \\ (M.Tech (CST), Central University of Punjab, Bathinda, India)
}

\begin{abstract}
A wireless sensor network consists of a large number of sensor nodes which are deployed over an area to perform local computations based on information gathered from the surroundings. Each node in the network consists of a battery, but it is very difficult to change or recharge batteries. So the question is how to enhance the lifetime of the network to such a long time. Therefore, in order to maximize the lifetime of the network, the consumption of energy must be minimized. This is an important challenge in sensor networks as sensors can not be easily replaced or recharged due to their ad-hoc deployment in hazardous environment. In this paper, the main techniques used for energy conservation in sensor networks are discussed which include duty cycling scheme, data driven approaches, mobility-based schemes, energy efficient MAC protocols and node self scheduling scheme. These schemes can be used to improve the energy efficiency of the wireless sensor network so that the network can work with greater efficiency and high battery lifetime.
\end{abstract}

Keywords : Energy conservation, energy consumption, sensor nodes, wireless sensor networks.

\section{INTRODUCTION}

A sensor network is a group of specialized transducers with a communications infrastructure intended to monitor and record conditions at diverse locations. Commonly monitored parameters are temperature, humidity, pressure, wind direction and speed, illumination intensity, vibration intensity, sound intensity, powerline voltage, chemical concentrations, pollutant levels and vital body functions [1].

\subsection{Sensor Network and its components}

A sensor network consists of multiple detection stations called sensor nodes, each of which is small, lightweight and portable [2]. It has the following components:

- Sensing subsystem: A sensing subsystem includes one or more sensors (with associated analog-to-digital converters) for data acquisition from the physical surrounding environment. It acts as a link to the outside world.

- Processing subsystem: It is used for local data processing and storage. It consists of a processor and memory.

- Wireless communication subsystem: It enables the sensor nodes to communicate with each other and with the base station. Basically, it is used for the transmission of data.

- Power Subsystem: A power source is required so that the device can perform various tasks uninterruptedly.

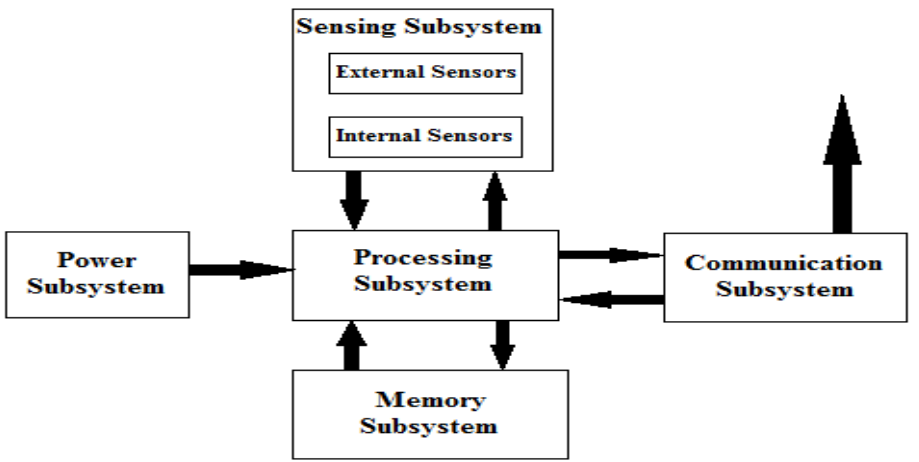

1.2 Sensor Node and its components

Fig. 1 Sensor Network Architecture

A sensor node is a tiny device which is equipped with a transducer, microcomputer, transceiver and power source [2].

- Transducer: The transducer generates electrical signals based on sensed physical effects and phenomena.

- Microcomputer: The microcomputer processes and stores the sensor output.

- Transceiver: The transceiver, which can be hard-wired or wireless, receives commands from a central computer and transmits data to that computer. 
- Power: The power for each sensor node is derived from the electric utility or from a battery.

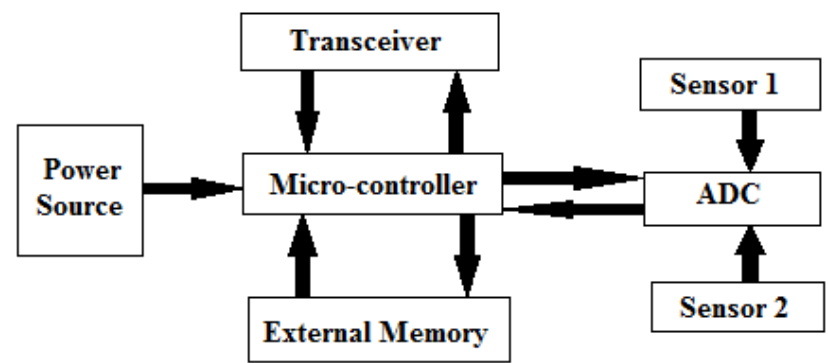

Fig. 2 Sensor Node

Energy conservation in sensor networks deals with reducing the consumption of energy among various intermediate nodes in the network so that the network life can be extended. A power source is required so that the device can perform various tasks uninterruptedly which usually consist of limited energy budget battery. Although it is easy to recharge the battery from time to time, but it may become impossible or inconvenient to recharge the battery in case if some sensor node is placed at very hostile or unpractical environment. The lifetime (in terms of months or years etc.) of sensor networks should be long enough to fulfill the application requirements. In some cases, energy can be scavenged from external environment e.g. solar power, but these resources are non-continuous in nature. So, energy conservation is the key issue in designing the systems based in wireless sensor networks.

\section{MAJOR SOURCES Of ENERGy WASTAGE In SENSOR NETWORKS}

In sensor networks, energy can be consumed either usefully or wastefully. Useful energy consumption includes sending and receiving data, processing and forwarding queries and data to other nodes. On the other hand, wasteful energy consumption may include the facts, like [4]

- Idle listening: It includes listening to an idle channel in order to receive possible traffic.

- Collision: The packets have to be resent after a collision has discarded the packets.

- Over-hearing: It includes receiving the packets that are destined to other nodes.

- Over-emitting: The packets that over-use the network are over-emitted.

\subsection{Issues in energy wastage in sensor network}

Energy in sensor networks is wasted in one or the other form. The major energy wastage occurs in the following subsystems as explained below.

2.1.1 Communication Subsystem: The communication subsystem has energy consumption much higher than the computation subsystem. It has been shown by experiments that transmitting one bit over the communication channel may consume as much as executing a few thousands instructions. Therefore, communication should be traded for computation.

2.1.2 Radio Consumption: The radio consumption is of the same order of magnitude in the reception (receiving the data), transmission (sending the data), and idle states (when no communication is taking place, i.e. no sending and receiving of data), while the power consumption drops of at least one order of magnitude in the sleep state. The sleep state of the node indicates that the node may generate packets but it will not transmit or receive any packet.

2.1.3 Sensing Subsystem: Depending on the specific application, the sensing sub-system might be another significant source of energy consumption, so its power consumption has to be reduced as well.

\section{ENERGY CONSERVATION IN SENSOR NETWORKS}

Based on the above issues, several approaches have to be exploited, even simultaneously, to reduce power consumption in wireless sensor networks. At a very general level, we identify five main enabling techniques, namely, duty cycling, data-driven approaches, mobility based schemes, energy efficient MAC protocols for sensor networks, and node self-scheduling scheme.

\subsection{Duty Cycling}

It is mainly focused on the networking subsystem. The most effective energy-conserving operation is putting the radio transceiver (transceiver that transmits and receives at the radio frequency, i.e. in the range of about $3 \mathrm{kHz}$ to $300 \mathrm{GHz}$ ) [3]) in the (low-power) sleep mode whenever communication is not required. In this way nodes alternate between active and sleep periods depending on the network activity. This behavior is usually referred to as duty cycling, and duty cycle is defined as the fraction of time nodes are active during their lifetime [2]. 
A sensor node has 4 operating modes: transmission, reception, idle listening and sleep. The most power consumption is due to transmission. Idle mode and receiving mode has approximately same power consumption. Sleep mode has the least power consumption. In duty cycling, the nodes that are currently not required can go to sleep and thus save energy. They need not to maintain the radio frequency regularly. Instead, they can switch off the radio when there is no network activity, thus alternating between sleep and wake-up periods [5].

\subsection{Data-driven approaches}

The goal of data driven approaches is to provide continuous data. It uses the concept of data sensing i.e. to sense the continuity of data. Data sensing impacts on energy consumption of the sensor nodes in two ways [2]:

- Unneeded samples: They lead to useless energy consumption. Sampled data generally has strong spatial and temporal correlation, so there is no need to communicate the redundant information to the sink.

- Power consumption: The power consumption of the sensing subsystem is not negligible .Energy wastage cannot be reduced only by reducing the communication.

Based on these two ways, data-driven approaches are divided into data reduction and energy efficient data acquisition schemes.

3.2.1 Data Reduction schemes: These schemes address the case of unneeded samples.

Data reduction can be divided into:

- In-network processing

- Data Prediction

- Data Compression

In-network processing: It consists in performing data aggregation (e.g., computing average of some values) at intermediate nodes between the sources and the sink. In this way, the amount of data is reduced while traversing the network towards the sink.

Data prediction: It consists in building an abstraction of a sensed phenomenon, for example a model describing data evolution. The model can predict the values sensed by sensor nodes within certain error bounds and reside both at the sensors and at the sink. If the needed accuracy is satisfied, queries issued by users can be evaluated at the sink through the model without the need to get the exact data from nodes.

Data compression: It is applied to reduce the amount of information sent by the source nodes.

\subsubsection{Energy-efficient data acquisition schemes}

They mainly focus on reducing the energy spent by the sensing subsystem. A sensor node's lifetime is fundamentally linked to the volume of data that it senses, processes and reports. Sensor Network allows environmental monitoring at extremely high spatial and temporal resolutions. But when raw data is to be extracted, certain problems arise, e.g. batteries may get drained due to excessive transceiver operation or data quality may deteriorate due to packet loss caused by congestion. To solve these problems, spatial correlation is maintained between the sensor nodes. In this method, instead of every node transmitting individually, a subset of nodes called correlating nodes are appointed that transmit the messages and act as representative of all the remaining nodes. It continues sending its own readings until a change in correlation is detected. When a change is encountered, it transmits an updated correlation message [2].

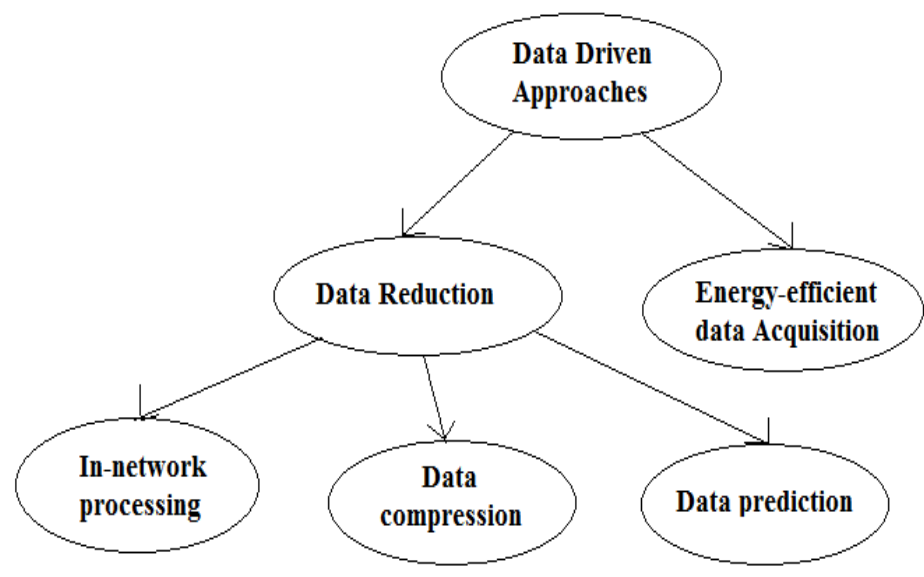

Fig. 3 Taxonomy of Data driven approaches 


\subsection{Mobility based schemes}

The mobility is defined as to describe the movement pattern of mobile nodes, location, velocity and acceleration change over time [4].Mobility based schemes define how the designer of the sensor network will control the mobility of the sensor nodes. Mobility can be random, predictable/deterministic or controlled. Mobility based schemes can be classified into two categories depending on the type of mobile entity:

- Mobile-sink schemes: Mobile-sink schemes include mobile sink nodes which offer transient shorter routes for relaying data, and offering connectivity to disconnected areas of network during any unfavorable situation, e.g. in case of fires, when some of the sensor nodes are destroyed due to fire, mobile sink nodes can provide connectivity with the other nodes of the network.

- Mobile-relay schemes: In this scheme, the relay stations can intelligently relay data between the base stations, i.e. the base station receiver automatically tunes on the base station transmitter and retransmits all signals received by the base station receiver. Mobile relays have the potential to provide performance improvements under high speed user mobility and heavy load conditions.

Mobile nodes can be divided into two broad categories. They can be specifically designed [2]

- As part of the network infrastructure: When they are part of the infrastructure, their mobility can be fully controlled and are, in general, robotized. Infrastructure refers to the architecture of the sensor network. When the mobile nodes are within the infrastructure, their movement can be controlled because they are still not transmitted over the network.

- As a part of the environment: When mobile nodes are part of the environment they might not be controllable. If they follow a strict schedule (e.g. strict space and timing relationship), then they have a completely predictable mobility (e.g., a shuttle for public transportation). Otherwise they may have a random behavior so that no reliable assumption can be made on their mobility. Finally, they may follow a mobility pattern that is neither predictable nor completely random. For example, this is the case of a bus moving in a city, whose speed is subject to large variation due to traffic conditions. In such a case, mobility patterns can be learned based on successive observations and estimated with some accuracy.

\subsection{Energy efficient MAC (Media Access Control) protocols for Sensor Networks}

Media access control (MAC) data communication protocol is a sub-layer of the data link layer. The MAC sub-layer provides addressing and channel access control mechanisms that make it possible for several terminals or network nodes to communicate within a multiple access network that incorporates a shared medium, e.g. Ethernet. Its functions generally include receiving and transmitting frames, appending, removing, padding the bits, etc.

Various types of MAC protocols used for energy conservation can be categorized as: contentionbased, TDMA-based and hybrid MAC protocols [8].

\subsubsection{Contention-based MAC protocols}

They are based on CSMA (Carrier Sense Multiple Access) or CSMA/CA (Carrier Sense Multiple Access/Collision Avoidance). It does not require any co-ordination among the nodes. Nodes will send the data and in case of collision, they will wait for a random duration of time before re-sending it again. The typical contention-based MAC protocols are S-MAC (Sensor-MAC) and T-MAC (Timeout-MAC) [5].

- S-MAC: There are two states in a time frame: active state and sleep state. S-MAC adopts an effective mechanism to solve the energy wasting problems, that is periodical listening and sleeping [6]. When a node is idle, it is more likely to be asleep instead of continuously listening to the channel. S-MAC reduces the listen time by letting the node go into periodic sleep mode.

- T-MAC: T-MAC is an extension of the previous protocol which adaptively adjusts the sleep and wake periods based on estimated traffic flow to increase the power savings and reduce delay [7]. TMAC also reduces the inactive time of the sensors compared to S-MAC. Hence, it is more energy efficient than SMAC.

\subsubsection{TDMA based MAC protocols}

They assign a unique time-slot for each node to send or receive data thereby making it collision-free. It avoids collisions and thus saves energy which would otherwise be wasted due to resending of data due to collisions and discarding [9].

\subsubsection{Hybrid contention based}

It has advantages of both contention based and TDMA-MAC protocols. It offers better scalability and flexibility and thus has high energy savings. 


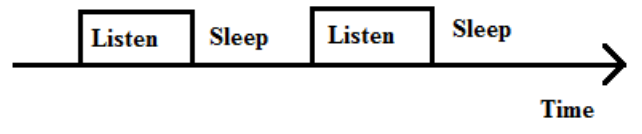

Fig. 4 Periodic listen and sleep

\subsection{Node Self-scheduling Scheme}

This technique is divided into two problems. The first problem is the rule that each node should follow to determine its work status. The second is the time when nodes should make such decision [10]. The assumptions of this work are as follows:

1) Each node knows its own location, which can be obtained at low cost from GPS or some localization system, and presumably is already available due to the needs of wireless sensor network applications. Also, each node knows who its neighbors are and where they are located, which is obtained through a simple neighbor information exchange process.

2) Each node knows the size of its sensing area. We assume that all nodes are deployed on a 2-dimensional plane and a node's sensing area is a circle centered at this node with known radius. The rule described there is also applicable to a 3-dimensional space.

3) The sensing range of each node can be identical or different. All the nodes in the network are timesynchronized.

4) After node-scheduling, some nodes are assigned off-duty state. Off-duty is a power saving sleep mode, compared to normal on-duty mode.

Off-duty may be implemented by following three ways:

- Powering off the sensing unit and communication unit

- Powering off the sensing unit

- Ignoring detected events without powering off any unit

Among the four methods, the first one consumes the least energy and the last one does the most. Therefore, the first one is the most desirable from the energy saving perspective. However, which method is used in practice depends on the intelligence owned by sensors and the cost for sleeping and waking up the sensing unit and communication unit [10].

The main objective of this scheme is to minimize the number of working nodes, as well as maintain the original sensing coverage. To achieve this goal, sensing area of each node is calculated and then compared with its neighbours'. If the sensing area of one node is fully embraced by the union set of its neighbours', i.e. neighbours can cover the current node's sensing area, this node falls asleep without reducing the system overall sensing coverage.

\section{CONCLUSION}

The emergence of wireless sensor networks can finally bridge the gap between physical and digital worlds. But the basic problem in sensor networks is the higher energy consumption. While transmitting data, a lot of energy is wasted in the various subsystems of the sensor network. For decreasing this loss of energy various methods are explained in this article. By implementing these methods, the wastage of energy in sensor networks can be reduced to a great extent. This will surely enhance the working of sensor network and hence greater outputs can be achieved from the same.

\section{REFERENCES}

[1] "Sensor Networks", available at: http://en.wikipedia.org/wiki/Wireless_sensor_network, last accessed on: 12 October, 2013.

[2] G. Anastasi, M. Conti, M. D. Francesco and A. Passarella, Energy conservation in wireless sensor networks: A survey, ELSEVIER, 2008, 537-568.

[3] "Radio Frequency", available at: http://en.wikipedia.org/wiki/Radio frequency, last accessed on: 15 th October, 2013.

[4] K. E. Kannammal and T. Purusothaman, Comparison of Data Centric Routing Protocols with Random Way Point Mobility Model in Mobile Sensor Networks, European Journal of Scientific Research, 65(4), 2011, 546.

[5] Z. Rezaei and S. Mobininejad, Energy Saving in Wireless Sensor, International Journal of Computer Science \& Engineering Survey (IJCSES), 3(1), 2012, 23-37.

[6] W. Ye, J. Heidemann and D. Estrin, Medium Access Control With Coordinated Adaptive Sleeping for Wireless Sensor Networks, IEEE/ACM Transactions on Networking, 12(3), 2004, 493-506.

[7] T. V. Dam and K. Langendoen, An Adaptive Energy-Efficient MAC Protocol for Wireless Sensor Networks, Proc. $1^{\text {st }}$ ACM Conf. on Embedded Networked Sensor Systems, Department of Computer Engineering Islamic Azad University, Arak Branch, Iran, 2003.

[8] W. Ye, J. Heidemann and D. Estrin, Medium Access Control With Coordinated Adaptive Sleeping for Wireless Sensor Networks, IEEE/ACM Transactions on Networking, 12(3), 2004, 493-506.

[9] T. V. Dam and K. Langendoen, An Adaptive Energy-Efficient MAC Protocol for Wireless Sensor Networks, Proc. 1st international conf. on Embedded networked sensor systems, Los Angeles, CA, USA, 2003, 171-180.

[10] D. Tian and N. D. Georganas, A Node Scheduling Scheme for Energy Conservation in Large Wireless Sensor Networks, Multimedia Communications Research Laboratory, University of Ottawa, Canada, 2003. 